\title{
Predictive diagnostics in colorectal cancer: impact of genetic polymorphisms on individual outcomes and treatment with fluoropyrimidine-based chemotherapy
}

\author{
Heidi Schwarzenbach
}

Received: 6 April 2010 / Accepted: 13 May 2010 / Published online: 4 June 2010

(C) European Association for Predictive, Preventive and Personalised Medicine 2010

\begin{abstract}
The 5-fluorouracil (5-FU)-based chemotherapy is a standard treatment for patients with colorectal cancer. However, a relevant number of patients suffer from severe toxic side effects, such as haemotoxicity, while lacking clinical response to adjuvant therapy. The inter-individual variations of drug toxicity and efficacy of the pyrimidine antagonist observed in clinical practice are mainly determined by genetic polymorphisms. The screening of genotypes, such as thymidylate synthase, dihydropyrimidine dehydrogenase, methylene tetrahydrofolate reductase, orotate phosphoribosyltransferase or glutathione Stransferase, could help identifying those patients with colorectal carcinoma who can actually benefit from a 5FU-based therapy. The current chapter elucidates the roles of the polymorphisms in the enzymes involved in the 5-FU metabolic pathway as prognostic and predictive markers. It reports on the relationship between various genotypes in patients with colorectal carcinoma and their responsiveness to a 5-FU-based chemotherapy, and concludes with an outlook on possible future directions in treatment of colorectal cancer.
\end{abstract}

Keywords Thymidylate synthase - Dihydropyrimidine dehydrogenase $\cdot$ Methylene tetrahydrofolate reductase . Orotate phosphoribosyltransferase - Glutathione S-transferase $\cdot$ Clinical outcome $\cdot$ Individualised treatment . Predictive markers

\footnotetext{
H. Schwarzenbach $(\bowtie)$

Institute of Tumour Biology,

University Medical Center Hamburg-Eppendorf,

Martinistrasse 52,

20246 Hamburg, Germany

e-mail: hschwarz@uke.uni-hamburg.de
}

\section{Introduction}

Colorectal tumours are a leading cause of cancer death worldwide. Surgical resection of primary and regional lymph nodes is among the standard therapies for treatment of colorectal tumours. Although surgical resection alone is potentially curative in colorectal cancer patients, many patients develop local or distant recurrences [1].

In the 1950s, fluoropyrimidines including 5-FU were introduced into clinical trials. Twenty years ago, adjuvant treatment of colon cancer patients was validated and improved the curative rates. Numerous analyses have shown that 5-FU-based systemic adjuvant chemotherapies have improved survival in high risk patients. In adjuvant settings, 5-FU-based chemotherapies are currently an integral component of the management of colon cancer. However, its clinical application is limited, because 5-FU has gastrointestinal and hematological toxicity. The additional introduction of active drugs, such as oxaliplatin, bevacicumab or cetuximab, has provided clinical benefit for the patient in both early and late stages of the disease. Adjuvant therapy for colorectal cancer patients consists primarily of combinations of 5-FU/leucovorin with oxaliplatin or oral capecitabine. The combined regimens of 5-FU, leucovorin and oxaliplatin have been shown to prolong significantly disease-free survival. In the treatment of colorectal cancer patients the introduction of the platinum compound oxaliplatin has increased response rates up to $25 \%$, even in heavily pretreated relapsing patients when administered in combination with 5-FU [2]. Also, the angiogenesis inhibitor bevacizumab and the epidermal growth factor receptor inhibitor cetuximab have shown activities when combined with 5-FU/leucovorin-based regimens as first-line treatment of advanced disease. For metastatic disease the 5-FU-based combination therapy with either irinotecan or oxaliplatin 
plus bevacizumab has resulted in a significant improvement in response, and disease-free and overall survival. However, a relevant number of patients still get toxic side effects from the treatment while lacking a response by the tumour. In the worst cases, chemotherapy toxicity may accelerate death [3]. Moreover, the risk of recurrence is still high in many patients. Therefore, there is a need to tailor the therapy. Predicting an effective regime with minimal toxicity in the palliative situation might potentially improve the quality of care by allowing individualization of treatment. The dose of agent and the combination of 5-FU with other drugs should be adapted for patients at high risk.

Even with different doses and schedules, the response rate of metastatic colorectal cancer is only $21 \%$ when treated with 5-FU and leucovorin. To predict the clinical outcome of colorectal cancer patients treated with adjuvant 5-FU-based chemotherapy, endeavours have been made to investigate the efficacy, tolerability and toxicity of 5-FU, together with why some subgroups do and other groups do not benefit from a 5FU-based therapy. Chemotherapy-based toxicity is caused by many factors such as organ dysfunction and genetic variability. In particular, polymorphic variations of the molecules involved in the metabolic pathway of 5-FU may contribute to the differences in clinical outcome among patients. Therefore, it is of note to investigate the genotypes and to generate a pharmacogenetic profile that may help predicting the clinical outcome of a 5-FU-based chemotherapeutic regimen. Several types of polymorphisms affect the gene expression of enzymes in the metabolism of 5-FU and include insertions, deletions, tandem repeats and single nucleotide polymorphisms. To choose the effective regime with minimal toxicity and to limit treatment failure, DNA derived from different tissues, such as tumour tissues, leukocytes and blood may be used for the experimental approaches on the genotypes. Associations between the potential drug toxicity and the genetic patterns may be determined by using germ-line DNA. Since the primary tumour harbours tumour-specific aberrations, which would otherwise escape analyses, the drug efficacy may be examined by DNA derived from the primary tumour.

\section{Polymorphisms of thymidylate synthase}

As shown in Fig. 1, the action mechanisms of the fluoropyrimidine 5-FU are the inhibition of thymidylate

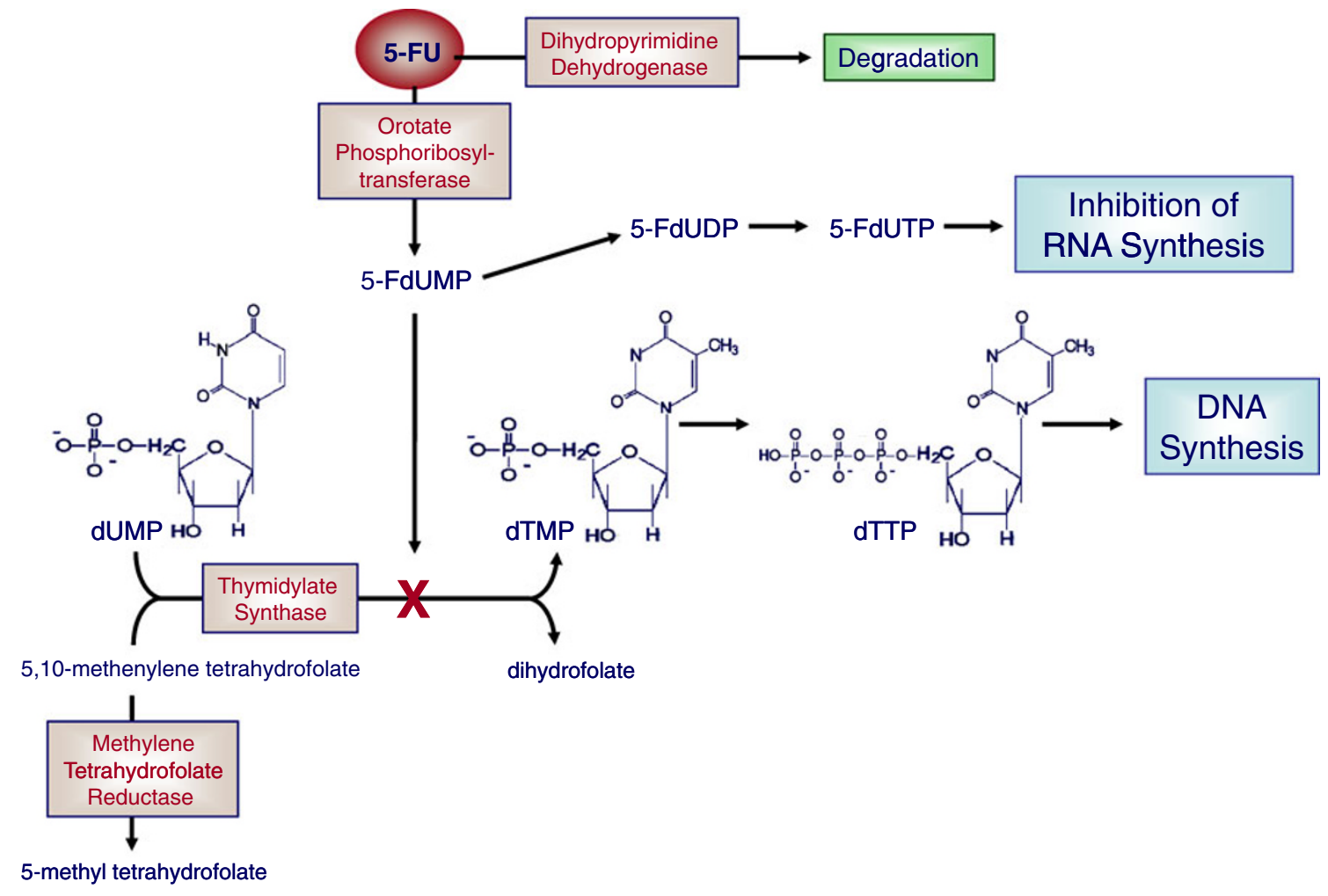

Fig. 1 The 5-fluorouracil (5-FU) metabolism pathway. In tumour cells 5-FU is converted to 5-fluorodeoxyuridine monophosphate (5-FdUMP) by the action of orotate phosphoribosyltransferase. 5-FdUMP inhibits the DNA synthesis by competing with deoxyuridine monophosphate (dUMP) for binding to thymidylate synthase in a complex that is stabilized by the reduced folate 5,10-methylene tetrahydrofolate. 5-FU can also inhibit RNA synthesis in a pathway that involves its metabolism by orotate phosphoribosyltransferase to 5-fluorouridine monophosphate (5-FUMP) and subsequent conversion to 5-fluorouridine triphosphate (5FUTP) via 5-fluorouridine diphosphate (5-FUDP). Extracellular catabolism of 5 -FU by dihydropyrimidine dehydrogenase reduces the level of 5-FU for the uptake into the tumour cells 
synthase (TS) by 5-FdUMP and the metabolic impairment of RNA by incorporation of 5-FdUTP into RNA [4]. Based on its polymorphisms and expression levels, TS has been described to be a predictive factor for the efficacy of the 5FU treatment $[5,6]$. As a key enzyme it regulates the dTMP levels by catalyzing the reductive methylation of dUMP by 5,10-methylene tetrahydrofolate. In this rate-limiting reaction, dTMP and dihydrofolate are formed. The thymidylate biosynthesis is the only de novo source for dTMP which is a precursor nucleotide for DNA replication. 5-FdUMP, an active metabolite of 5-FU binds to TS, and causes the formation of a stable ternary complex which suppresses the enzyme activity of TS [7]. As a result, dUMP molecules accumulate along with a decrease in dTMP levels. After conversion of dUMP into dUTP, dUTP replaces dTTP as a substrate for DNA polymerases and is incorporated into the newly synthesized DNA strand. Uracyl-N-glycosylase actually detects incorrectly incorporated uracil bases in the DNA strand, e.g. after the spontaneous deamination of deoxycytidine. Although it removes the incorporated uracil bases, the new DNA strand is incorrectly repaired by dUTP or 5-FdUTP, because dTTP is not available.

Since TS is required for the delivery of nucleotides used in DNA replication, and consequently, for tumour cell proliferation and tumour growth, numerous reports have engaged in studying the polymorphisms of TS gene, and demonstrated that the intratumoural yields of TS mRNA transcripts and protein molecules may be a predictor of response and survival to 5-FU-based chemotherapy [8-10]. Whereas low TS mRNA levels may associate with a better response to 5-FU-based chemotherapy and improve 5-year survival, higher expression levels may fail to respond to this treatment $[11,12]$. However, the minor TS levels may also enhance the cytotoxic side effects of 5-FU provoking the determination of patient therapy [9, 13]. These observations suggested that screening of TS genotype that is linked with TS expression might have the potential to identify those patients with colorectal carcinoma who benefit from a 5-FU-based adjuvant therapy. So far, three functional polymorphisms of the TS gene have been identified: variable number tandem repeat (VNTR), single nucleotide (SNP) and deletion (1494del6) polymorphisms, which significantly affect TS gene expression.

With respect to the VNTR polymorphism, the 5' regulatory region of the TS promoter contains a variable number of a 28 -bp repeat (R), mainly, double (2R) or triple $(3 R)$ repeats. These tandem repeats contain E-boxes for the transcription factor USF-1 (upstream stimulatory factor-1) that activate transcription of the TS gene by binding to these motifs [14]. Accordingly, the higher number of repeats is associated with a higher promoter activation and transcription. It was reported that of the three VNTR groups, including $2 \mathrm{R} / \mathrm{loss}, 2 \mathrm{R} / 3 \mathrm{R}$ and $\operatorname{loss} / 3 \mathrm{R}$, the poly- morphism $2 \mathrm{R} /$ loss exhibited the lowest mRNA expression level, the best response to 5-FU and the longest medium survival time [15].

A further polymorphism is a $\mathrm{G} / \mathrm{C}$ (guanine/cytosine) substitution of the 12th nucleotide in the second repeat of the triple repeat variant $(3 R C / 3 R G)$. The presence of the SNP in the USF-1 binding site causes a decrease in transcriptional activity of the wild type triple repeat $(3 \mathrm{RG} / 3 \mathrm{RG})$ variant which is comparable with the $2 \mathrm{R} / 3 \mathrm{RG}$ variant [16]. Functional analysis showed that a plasmid construct encoding for the 3RG/3RG variant had 3-4 times higher translation efficiency than other polymorphic sequences [17].

In addition, a 6-bp deletion (1494del6) polymorphism was identified in the $3^{\prime}$ untranslated transcriptional region (3'UTR) of the TS gene. An association between this deletion (del) and the mRNA instability has been described [18]. To investigate the function of the 1494del6 polymorphism in vitro, a luciferase system was used, and showed a $\sim 70 \%$ decrease in luciferase activity and mRNA levels. The TS 3'UTR construct containing the heterozygous del/6 bp deletion also had a higher rate of mRNA degradation compared to the wild type (6 bp/6 bp) construct [18]. As examined in studies by Kawakami et al. [19] and Uchida et al. [20], the deletion at the TS locus in tumour cells may affect the actual expression level. It seems that TS promoter and 3'UTR polymorphisms are dependent on one another, because disequilibrium between both polymorphisms may be a general feature of the TS gene [21,22]. Conversely, Merkelbach-Bruse et al. disproved an association between microsatellite instability (MSI) status and TS expression. Based on a higher mRNA stability, they observed that the $3 R / 3 R$ or $2 R / 3 R$ genotypes of TS had higher mRNA levels whereas polymorphic variants of the $3^{\prime} \mathrm{UTR}$ did not influence the TS mRNA level [23].

The TS gene is localized on the short chromosomal arm 18. Vogelstein et al. reported that $\sim 40 \%$ of patients with colorectal tumours had deletions in this region [24]. Other laboratories even found losses of heterozygosity $(\mathrm{LOH})$ in more than half of the examined carcinomas [25]. These patients possess an imbalance of the TS allele that remains occult on examining exclusively the genotype of the leukocyte (wild type) DNA. Frequent $\mathrm{LOH}$ at the TS locus was detected in $62 \%$ of the informative cases by RT-PCR using TS mRNA and microsatellite analyses of the marker D18S59 located downstream of the TS gene sequence [19]. These high frequencies of DNA losses support an unstable TS genotype during carcinogenesis. It is of note that in respect of the repeat number of the heterozygous genotype $(3 \mathrm{R} / 2 \mathrm{R})$ bearing $\mathrm{LOH}$, the cancer tissue with the genotype $2 \mathrm{R} /$ loss expressed a significantly lower level of TS protein than did the tumour with the genotype $3 \mathrm{R} /$ loss [19]. These data indicate a significant difference between the TS genotypes of tumour and normal tissues. 
In respect to the different TS variants, discrepancies in risk of toxicity, clinical outcome and gene expression have been reported, because most studies have considered only a single TS polymorphism, [16]. Moreover, it should be critically noted that genotyping of the TS variants in numerous analyses was performed by using normal leukocyte DNA instead of tumour DNA indicating that the occurrence of LOH was not considered [26]. A recent study explored the effect of the three polymorphisms of the TS gene on overall and progression-free survival of colorectal cancer patients subjected to 5-FU-based chemotherapy and showed that the $3 R G / 3 R G 6 \mathrm{bp} / 6 \mathrm{bp}$ combined genotype (wild type, high expression) identified a subgroup of advanced patients with poor prognosis. However, none of the studied polymorphisms alone affected overall or progression-free survival [27]. A further study investigated the three polymorphisms of the TS gene and the response to a 5-FU-based chemotherapy in patients with colorectal cancer and liver metastases. The distinction between high $(2 \mathrm{R} / 3 \mathrm{G}, 3 \mathrm{RC} / 3 \mathrm{RG}$ and $3 \mathrm{RG} / 3 \mathrm{RG})$ and low $(2 \mathrm{R} / 2 \mathrm{R}, 2 \mathrm{R} /$ $3 R C$ and $3 R C / 3 R C$ ) $T S$ expression genotypes referred to a significant association with tumour response [28]. Tumour recurrence is a major problem after curative resection in the management of colon cancer therapy. Lurje et al. showed that high-expression variants of TS may help to identify stage II and stage III colon cancer patients who are at high risk of developing tumour recurrence, and also those who are more likely to benefit from a 5-FU-based adjuvant chemotherapy. Patients harbouring the TS 3RG $6 \mathrm{bp}$ haplotype were at highest risk in developing tumour recurrence. In both univariate and multivariable analyses, TS 3RG alone or in combination with TS 1494del6 proved to be adverse prognostic markers [29].

Numerous reports have not only argued for, but also against, the incidence of diverse polymorphisms occurring with a better clinical response and overall survival of the patients [9, 30-32]. A recently published research showed that the different polymorphisms of the TS gene (SNP and VNTR) may not be related with disease-free survival in patients with stage III colorectal cancer receiving adjuvant chemotherapy [33]. In our study, we investigated whether the presence of single polymorphisms (VNTR, 1494del6 and SNP), LOH at the microsatellite markers D18S59, D18S1140 and D18S976 mapped up- and downstream to the TS locus, and combinations of them at the TS locus in leukocytes, primary tumour and blood of patients with advanced colorectal carcinoma who received 5-FU-based chemotherapy, as well as their increased DNA concentrations in blood, were associated with the clinical and histopathological risk parameters of these patients. Apart from the significant correlations of the cell-free serum DNA concentrations with the $1494 \mathrm{del} 6$ and combined polymorphisms, and of the age with VNTR and VNTR plus 1494del6, the combined polymorphisms detected in blood serum only tended to associate with the response to therapy by the patients. Moreover, the borderline significance detected in serum showed rather an inverse correlation to the number of polymorphisms and a low impact on response to 5-FU. Our findings provided an insight into the molecular diversity of the regulation of TS gene expression, and suggested that the variance in responses to 5-FU-based chemotherapy cannot consistently be explained by the genetic alterations detected in the TS gene [34]. Although numerous in vivo and in vitro studies have described the association between TS genotype with TS protein level $[9,14,17]$, a recent report could not find such correlations by immunohistochemistry. This laboratory also showed that the immunohistochemistry data did not significantly associate with the clinical outcome of patients, and that VNTR was the only polymorphism of the TS gene demonstrating a significant association with later recurrence and cancer-specific survival. Assessment of the two other polymorphisms (SNP and 1494del6) did not deliver any additionally prognostic information [35].

Summing up, these studies show that the prognostic value of TS genotypes in patients receiving adjuvant 5-FUbased chemotherapy is still a matter of controversy. They also reflect the complexity of TS gene regulation and that other enzymes involved in thymidylate biosynthesis should also be considered.

\section{Polymorphisms of orotate phosphoribosyltransferase}

As shown in Fig. 1, 5-FU is preferentially phosphorylated by the orotate phosphoribosyltransferase (OPRT) to produce the nucleotide 5-FdUMP. 5-FdUMP is then phosphorylated to 5-FdUDP, which can be either reconverted to 5-FdUMP or phosphorylated to the active nucleotide 5-FdUTP. Now, 5FdUTP can be extensively incorporated into RNA (F-RNA), disrupting normal RNA process and function [36]. An inhibitor of OPRT is potassium oxonate which could lower the levels of 5-FdUMP leading to a decrease of $~ 70 \%$ in FRNA synthesis in the small intestine and a reduction in gastrointestinal toxicity [37].

In mucosal carcinoma and infiltrative lesions the expression of OPRT correlated significantly with the presence of lymphovascular invasion suggesting that OPRT is involved in the invasion and metastasis of colorectal tumours [38]. On the other hand, using immunohistochemistry, Tokunaga et al. found that a high OPRT expression in stages II-IV disease was correlated with a better overall survival [39]. Common polymorphisms, including SNPs 213G/C (guanine/cytosine substitution at position 213),638G/C, 1050T/A (thymine/ adenine) and $1336 \mathrm{~A} / \mathrm{G}$ (adenine/guanine), have been identified in the coding region of OPRT [40]. The SNP $213 \mathrm{G} / \mathrm{C}$ of OPRT may be a significant predictor of 5-FU-induced 
toxicity in patients treated with 5-FU adjuvant therapy, and have an independent value for prediction of severe grade 3 to 4 diarrhea [41]. In contrast, the SNPs 638G/C, 1050T/A, and $1336 \mathrm{~A} / \mathrm{G}$ of OPRT may have no major influence on $5-\mathrm{FU}$ sensitivity [42].

\section{Polymorphisms of dihydropyrimidine dehydrogenase}

The systemic exposure to 5-FU is fundamentally determined by the genetically polymorphic enzyme dihydropyrimidine dehydrogenase (DPD). DPD is the initial and rate-limiting enzyme in the three-step catabolic pathway of 5-FU (Fig. 1). More than $80 \%$ of administered $5-\mathrm{FU}$ is degraded by DPD in human liver. Deficiency in the DPD enzyme activity caused a delay in the clearance of 5-FU from blood and the accumulation of 5-FU [43]. As a result, chemotherapeutic toxicity of 5-FU has been associated to low levels of DPD enzyme activity. At least $50 \%$ of patients who had highly toxic side effects were genotypically heterozygous or homozygous for SNPs in the DPD gene [44, 45].

As determined by immunohistochemistry and/or RTPCR, analyses on DPD expression in primary colorectal tumours of patients treated with 5-FU-based chemotherapy showed either significant associations of a low DPD expression with prolonged overall and disease-free survival $[46,47]$ or no associations [48]. Accordingly, immunohistochemistry of paraffin-embedded primary tumour tissues demonstrated that patients with high DPD expression levels had significantly shorter disease-free and overall survival than patients with low DPD levels. In combined analyses, including the determination of TS and DPD expression, patients with low TS and DPD had the best outcome in terms of disease-free and overall survival. This retrospective study suggested that the combined assessment of TS and DPD might be useful in evaluating the prognosis of patients with Dukes' B and C colon carcinoma receiving 5-FU adjuvant chemotherapy [46]. However, conflicting results have been reported in metastatic patients [39, 49, 50].

Although the precise role of DPD as a prognostic factor in the efficacy of 5-FU still has to be established, there is evidence that patients with a DPD deficiency are prone to the development of severe 5-FU-associated toxicity. The exon 14-skipping mutation (IVS14+1G/A mutation) provokes a changed splice site leading to an aberrant protein sequence and a loss of the catalytic activity of DPD. This gene variant accounted for $24-28 \%$ of all patients suffering from severe 5-FU toxicity [51]. In the cofactor binding domain and the electron transport domain of DPD the 2846A/T polymorphism leads to an amino acid substitution and a reduced enzyme activity. This gene variant has been detected in $2 \%$ of the population [52]. To tailor 5-FU administration and determine strategy in first-line chemo- therapy of advanced colorectal cancer, Capitain et al. investigated the polymorphisms of DPD and their influence on tolerance and toxicity in patients initially treated with 5FU. They detected that the toxicity was significantly linked to the SNP 2846A/T and the splicing mutation IVS14+1G/ A of DPD [53]. In five out of eight cases with severe side effects, Amstutz et al. observed that a potential association existed between a haplotype containing three intronic polymorphisms (IVS5+18G/A, IVS6+139G/A and IVS9+51 T/G) and a SNP (1236G/A). Comparing their data with those from other studies, they suggested that the relative importance of particular DPD mutations (IVS14+1G/A and 2846A/T) for predicting severe 5-FU toxicity differs geographically across Europe [54]. Moreover, numerous further polymorphisms in the DPD gene, such as $85 \mathrm{~T} / \mathrm{C}, 496 \mathrm{~A} / \mathrm{G}, 1601 \mathrm{G} / \mathrm{A}$ and $1627 \mathrm{~A} / \mathrm{G}$, have been identified but their effect on transcription and their influence on clinical outcome of colorectal cancer patients treated with 5-FU are still unclear [55].

\section{Polymorphisms of methylene tetrahydrofolate reductase}

5,10-methylene tetrahydrofolate reductase (MTHFR) is a key enzyme in the regulation of intracellular folate levels. It affects both, DNA synthesis and methylation. In a onecarbon metabolism it catalyses the irreversible conversion of 5,10-methylene tetrahydrofolate to 5-methyl tetrahydrofolate to generate the folate derivative for homocysteine remethylation to methionine (Fig. 1). In human cancer cells the inhibition by specific antisense RNA molecules against MTHFR affected tumour growth, and 5-FU could potentiate this significant inhibitory effect [56].

Two common SNPs (677C/T and 1298A/C) have been described to reduce MTHFR enzyme activity and contribute to an alteration of intracellular folate distribution. The $677 \mathrm{C} / \mathrm{T}$ transition in exon 4 results in an alanine to valine substitution in the predicted catalytic domain of MTHFR. This substitution made the enzyme thermolabile, and reduced $\sim 70 \%$ and $\sim 35 \%$ of its enzyme activity in homozygotes and heterozygotes, respectively [57]. The $1298 \mathrm{~A} / \mathrm{C}$ transition in exon 7 results in a glutamate to alanine substitution within the regulatory domain of the protein and led to a decreased enzyme activity, albeit not to the same extent as that of the $677 \mathrm{C} / \mathrm{T}$ allele [58]. Since a decrease in MTHFR enzymatic activity may lead to a decrease in intracellular 5-methyl tetrahydrofolate concentrations, it is presumed that tumours exhibiting the rare MTHFR variants may be more sensitive to 5-FU cytotoxicity than patients with a wild-type genotype. Moreover, the concomitantly higher concentrations of 5,10-methylene tetrahydrofolate may in turn favour the formation and stability of the inhibitory ternary complex consisting of TS, 5,10-methylene tetrahydrofolate and 5-FdUMP. 
Several clinical studies have investigated the potential predictive role of these genetic variants in toxicity and efficacy of 5-FU, but contradictory data have been published [59]. Fernandez-Peralta et al. examined the relationship of $677 \mathrm{C} / \mathrm{T}$ and $1298 \mathrm{~A} / \mathrm{C}$ polymorphisms with biological, clinicopathological, genetic and epigenetic features of the colorectal tumours and showed that both polymorphisms were relevant and independent factors of patient outcome after 5-FU-based treatment. The variant $677 \mathrm{C} / \mathrm{T}$ had a protective effect on colorectal cancer development, whereas the variant $1298 \mathrm{~A} / \mathrm{C}$ did not produce any effect on disease risk [60]. Moreover, both polymorphisms were reported to be associated with a sexspecific clinical outcome in metastatic colon cancer patients treated with FU-based chemotherapy. The 1298A/C polymorphism in MTHFR correlated statistically significantly with the overall survival of female patients with metastatic colon cancer, but no such correlation was observed for male patients. Therefore, it was suggested that this variant might be a prognostic marker for female patients with metastatic colon cancer [61]. These findings have been supported by another study showing that the overall survival was higher for $\mathrm{C} / \mathrm{C}$ female patients than for $\mathrm{A} / \mathrm{C}$ female patients [62]. Etienne et al. evaluated the MTHFR polymorphisms in patients with metastatic colorectal cancer who were treated with 5-FU and leucovorin. Their response rate was significantly associated with the $677 \mathrm{C} / \mathrm{T}$ genotype, but not connected with the 1298A/C genotype [63]. In the study by Jakobsen et al. the response rate was $66 \%$ for $677 \mathrm{~T} / \mathrm{T}$ compared with $33 \%$ and $21 \%$ for $677 \mathrm{C} / \mathrm{C}$ and $677 \mathrm{C} / \mathrm{T}$, respectively. No correlation was observed in the case of $1298 \mathrm{~A} / \mathrm{C}$ polymorphism [64]. In contrast, the $677 \mathrm{C} / \mathrm{T}$ polymorphism was not associated with the clinical response of metastatic colorectal cancer patients who received 5-FU/ oxaliplatin/leucovorin chemotherapy and caused severe grade 3 to 4 diarrhoea [65]. Although the variant $677 \mathrm{C} / \mathrm{T}$ did not affect the risk of colorectal cancer, it affected the sensitivity to chemotherapy and the risk of side-effects and therefore survival in stage III and possibly stage IV colon cancer [66]. It was also reported that patients initially treated with 5-FU had a severe toxicity when they were homozygous carriers of 1298C/C [67].

As pointed by out several studies [68-70] and documented by the above-described data, SNPs in the MTHFR gene cannot be considered as independent factors of outcome in colorectal cancer patients treated with 5-FU-based chemotherapy.

\section{Polymorphisms of glutathione S-transferase}

Glutathione S-transferases (GSTs) are members of a superfamily of dimeric phase II detoxifying enzymes. They protect cellular macromolecules from damage by catalyzing the conjugation of toxic and carcinogenic electrophilic molecules with glutathione. The resulting complex is less toxic and more readily excreted. In contrast to the other members of the family, such as GSTT1, GSTM1 and GSTA1, the variant GSTP1 is predominantly expressed in colon. Diverse common SNPs have been described for the GST genes that either abolish or decrease or increase the enzyme activity of GSTs for electrophilic substances [67].

To predict the toxicity in metastatic colorectal cancer patients treated with 5-FU/irinotecan/leucovorin regimens, the roles of $69 \mathrm{C} / \mathrm{T}$ in GSTA1, 105Ile/Val (isoleucine/valine substitution) in GSTP1, and null allele (homozygous DNA deletion) in GSTT1 and GSTM1 were evaluated. In human tissues a decline in GSTP1 activity was reported to parallel with an increasing number of 105Ile/Val alleles in GSTP1 [71]. Thus, a valine residue at position 105 of the GSTP1 protein resulted in decreased enzyme activity. A strongly significant association was observed between the null allele in GSTT1 and gastrointestinal toxicity. In comparison to $23 \%$ of patients with an intact GSTT1 genotype, $57 \%$ of null allele carriers developed gastrointestinal grade 3 toxicity. Thus, patients with a null allele in GSTT1 had a greater probability of developing toxicity to 5-FU/irinotecan/ leucovorin than patients who lacked this deletion. These findings indicated that the null allele in GSTT1 gene might be considered as a new marker when the treatment schedule is administered to metastatic colorectal cancer patients. Besides, the other polymorphisms analysed in that study did not refer to toxicity [72]. In addition, the neurotoxicity of patients with advanced colorectal cancer treated with a chemotherapy of 5-FU, oxaliplatin and folinic acid was reported to be significantly associated with the 105Ile/Val polymorphism in GSTP1. Carriers of the 105Val/Val genotype were more prone to suffer from grade 3 neurotoxicity than carriers of 105Ile/Val and 105Ile/Ile genotypes [67].

To predict the clinical outcome of 5-FU/oxaliplatinbased chemotherapy in patients with advanced colorectal cancer and refractory stage IV disease, the SNP 105Ile/Val in GSTP1 was examined. Stoehlmacher et al. demonstrated that the $105 \mathrm{Ile} / \mathrm{Val}$ variant might be a predictor for time to progression to 5-FU/oxaliplatin, as well as overall survival. They suggested that patients with this variant allele may experience protracted detoxification of oxaliplatin, and that a prolonged exposure of the tumour to oxaliplatin may lead to an increased overall efficacy of the drug and a superior survival of the patients. In contrast, the null alleles of GSTT1 and the GSTM1 linked to an abolished enzyme activity had no effect on clinical outcome [73]. Moreover, the same laboratory showed that the 105Ile/Val polymorphism in GSTP1 was associated in a dose-dependent fashion with increased survival of patients with advanced 
colorectal cancer receiving 5-FU/oxaliplatin chemotherapy. Patients with the genotypes 105Val/Val, 105Ile/Ile and $105 \mathrm{Ile} / \mathrm{Val}$ had a median survival of $24.9,7.9$ and 13.3 months, respectively [74].

Although the published data are contradictory, they underline the fact that GST genotyping of individual colorectal cancer patients receiving 5-FU-based chemotherapy may contribute to improved therapy planning.

\section{Interactions between the different genotypes}

Some studies tried to go beyond the analyses of single genetic polymorphisms to a more comprehensive investigation that identifies genomic variants and patterns of several polymorphisms. However, also these investigations showed contradictory data, thus leading to increasing uncertainty in the identification of reliable predictive genetic markers of drug response. The interactions between ten candidate polymorphisms of nine genes and the incremental toxicity of adding irinotecan or oxaliplatin to FU were investigated in a large randomized trial. Among the different putative pharmacogenetic markers assessed, the polymorphisms of TS, DPD, MTHFR and GSTP1 were also analyzed. However, none of the polymorphisms was significantly associated with the toxicity of any regimen and the primary outcome [75]. Moreover, six gene polymorphisms, among others of GSTP1, MTHFR and TS, were assessed in Taiwanese patients who were diagnosed at high-risk stages and administered adjuvant chemotherapy regimens that included 5-FU and leucovorin. Also in this study, no correlations between genetic polymorphisms and clinicopathological features of the patients were detected [76]. However, Capitain et al. who investigated different germinal polymorphisms and 5-FU plasma clearance in patients of advanced colorectal cancer initially treated by 5 FU reported that the overall survival of the patients with a 3R/3R genotype of TS associated with the homozygosity of $\mathrm{C} / \mathrm{C}$ for $677 \mathrm{C} / \mathrm{T}$ or $\mathrm{A} / \mathrm{A}$ for $1298 \mathrm{~A} / \mathrm{C}$ of MTHFR was statistically shorter [53].

These data show that there is a considerable need to increase efforts in the screening of several polymorphic variants in the 5-FU metabolic pathway to improve clinical outcome in the treatment of colorectal cancer,

\section{Conclusions}

The pharmacogenetics of drug-metabolizing systems deals with the identification of genetic polymorphisms that are responsible for inter-individual variation in the response to drug therapy. In the current chapter, pharmacogenetic studies investigating the drug targets of the 5-FU metabo- lism pathway in the treatment of colorectal cancers have been discussed. Several enzymes are involved in the catabolism and anabolism of 5-FU, and their activity varies largely between individuals. As a result the anti-tumour activity of 5-FU is limited by the genetic polymorphisms of these enzymes.

Inconsistent data on the relationship of the diverse variants of the thymidylate synthase, dihydropyrimidine dehydrogenase, methylene tetrahydrofolate reductase, orotate phosphoribosyltransferase and glutathione S-transferase to a better clinical outcome and survival of the patients, and to response to drug toxicity have been published. To date, this discrepancy underscores the complexity of the 5-FU metabolic pathway. Since several enzymes with diverse polymorphisms are involved in this pathway, it is questionable to only examine the polymorphisms of one enzyme and to ignore the impact of the genotypes of the other enzymes on the clinical outcome of the patients. In addition, the contradictory data amongst the various studies may be due to retrospective analyses and relatively small patient cohorts. Further reasons may be the performance of different techniques, such as immunohistochemistry and RT-PCR, which have been used to examine the expression of these enzymes, the genotyping in normal or tumour tissues, missing PCR-based microsatellite analyses for the evaluation of $\mathrm{LOH}$, variable doses and schedules of 5-FUbased chemotherapy and variable tumour stages of the patients.

To enhance therapeutic effect and to minimize toxicity of 5-FU-based chemotherapies, it is important to gain a better understanding of the metabolism of 5-FU agents and to scrutinize which molecular determinants exert a dominating influence on the response to 5-FU. To advance 5-FU-based chemotherapies, multivariable approaches on diverse molecular markers and prospective clinical trials with large patient cohorts displaying statistical power are required. The identification of relevant germ-line polymorphisms as well as tumour-specific somatic mutations, combined with their mRNA or protein expression levels in tumour tissue, might permit more effective and individualised 5-FU-based chemotherapy in future. Selective genotypical and phenotypical information should facilitate the performance of individualised chemotherapy regimen and combinations of chemotherapeutic agents with the adequate doses of drugs. Technologies, such as microarray and proteomics, may lead to the identification of predictive markers and should improve the further development of patient treatment. Finally, for improving the outlook of patients with colorectal cancer, a number of new agents (e.g., capecitabine eniluracil, tomudex) targeted to the enzymes in 5-FU pathway have been synthesized, and novel combinations of 5-FU or its analogs with agents (e.g., oxaliplatin, irinotecan) have been examined. 


\section{References}

1. Stohlmacher J. Pharmacogenetics in gastrointestinal tumors. Onkologie. 2005;28:435-40.

2. Bleiberg H. Role of chemotherapy for advanced colorectal cancer: new opportunities. Semin Oncol. 1996;23:42-50.

3. Rothenberg ML, Meropol NJ, Poplin EA, Van Cutsem E, Wadler S. Mortality associated with irinotecan plus bolus fluorouracil/ leucovorin: summary findings of an independent panel. J Clin Oncol. 2001;19:3801-7.

4. Spears CP, Gustavsson BG, Mitchell MS, Spicer D, Berne M, Bernstein L, et al. Thymidylate synthetase inhibition in malignant tumors and normal liver of patients given intravenous 5fluorouracil. Cancer Res. 1984;44:4144-50.

5. Gibson TB. Polymorphisms in the thymidylate synthase gene predict response to 5-fluorouracil therapy in colorectal cancer. Clin Colorectal Cancer. 2006;5:321-3.

6. Nief N, Le Morvan V, Robert J. Involvement of gene polymorphisms of thymidylate synthase in gene expression, protein activity and anticancer drug cytotoxicity using the NCI-60 panel. Eur J Cancer. 2007;43:955-62.

7. Iacopetta B. Methyl-group metabolism and the response of colorectal cancer to 5-fluorouracil. Crit Rev Oncog. 2006;12:11526.

8. Peters GJ, Backus HH, Freemantle S, van Triest B, CodacciPisanelli G, van der Wilt CL, et al. Induction of thymidylate synthase as a 5-fluorouracil resistance mechanism. Biochim Biophys Acta. 2002;1587:194-205.

9. Pullarkat ST, Stoehlmacher J, Ghaderi V, Xiong YP, Ingles SA, Sherrod A, et al. Thymidylate synthase gene polymorphism determines response and toxicity of 5-FU chemotherapy. Pharmacogenomics J. 2001;1:65-70.

10. Morganti M, Ciantelli M, Giglioni B, Putignano AL, Nobili S, Papi L, et al. Relationships between promoter polymorphisms in the thymidylate synthase gene and mRNA levels in colorectal cancers. Eur J Cancer. 2005;41:2176-83.

11. Leichman CG, Lenz HJ, Leichman L, Danenberg K, Baranda J, Groshen S, et al. Quantitation of intratumoral thymidylate synthase expression predicts for disseminated colorectal cancer response and resistance to protracted-infusion fluorouracil and weekly leucovorin. J Clin Oncol. 1997;15:3223-9.

12. Shirota Y, Stoehlmacher J, Brabender J, Xiong YP, Uetake H, Danenberg KD, et al. ERCC1 and thymidylate synthase mRNA levels predict survival for colorectal cancer patients receiving combination oxaliplatin and fluorouracil chemotherapy. J Clin Oncol. 2001;19:4298-304.

13. Yawata A, Kim SR, Miyajima A, Kubo T, Ishida S, Saito Y, et al. Polymorphic tandem repeat sequences of the thymidylate synthase gene correlates with cellular-based sensitivity to fluoropyrimidine antitumor agents. Cancer Chemother Pharmacol. 2005;56:465-72.

14. Mandola MV, Stoehlmacher J, Muller-Weeks S, Cesarone G, Yu $\mathrm{MC}$, Lenz $\mathrm{HJ}$, et al. A novel single nucleotide polymorphism within the $5^{\prime}$ tandem repeat polymorphism of the thymidylate synthase gene abolishes USF-1 binding and alters transcriptional activity. Cancer Res. 2003;63:2898-904.

15. Ott K, Vogelsang H, Marton N, Becker K, Lordick F, Kobl M, et al. The thymidylate synthase tandem repeat promoter polymorphism: a predictor for tumor-related survival in neoadjuvant treated locally advanced gastric cancer. Int J Cancer. 2006;119:288594.

16. Gusella M, Padrini R. G $>$ C SNP of thymidylate synthase with respect to colorectal cancer. Pharmacogenomics. 2007;8:985-96.

17. Kawakami K, Watanabe G. Identification and functional analysis of single nucleotide polymorphism in the tandem repeat sequence of thymidylate synthase gene. Cancer Res. 2003;63:6004-7.
18. Mandola MV, Stoehlmacher J, Zhang W, Groshen S, Yu MC, Iqbal S, et al. A 6 bp polymorphism in the thymidylate synthase gene causes message instability and is associated with decreased intratumoral TS mRNA levels. Pharmacogenetics. 2004;14:31927.

19. Kawakami K, Ishida Y, Danenberg KD, Omura K, Watanabe G, Danenberg PV. Functional polymorphism of the thymidylate synthase gene in colorectal cancer accompanied by frequent loss of heterozygosity. Jpn J Cancer Res. 2002;93:1221-9.

20. Uchida K, Hayashi K, Kawakami K, Schneider S, Yochim JM, Kuramochi $\mathrm{H}$, et al. Loss of heterozygosity at the thymidylate synthase (TS) locus on chromosome 18 affects tumor response and survival in individuals heterozygous for a 28-bp polymorphism in the TS gene. Clin Cancer Res. 2004;10:433-9.

21. Dotor E, Cuatrecases M, Martinez-Iniesta M, Navarro M, Vilardell F, Guino E, et al. Tumor thymidylate synthase 1494del6 genotype as a prognostic factor in colorectal cancer patients receiving fluorouracil-based adjuvant treatment. J Clin Oncol. 2006;24:1603-11.

22. Lecomte T, Ferraz JM, Zinzindohoue F, Loriot MA, Tregouet DA, Landi B, et al. Thymidylate synthase gene polymorphism predicts toxicity in colorectal cancer patients receiving 5-fluorouracilbased chemotherapy. Clin Cancer Res. 2004;10:5880-8.

23. Merkelbach-Bruse S, Hans V, Mathiak M, Sanguedolce R, Alessandro R, Ruschoff J, et al. Associations between polymorphisms in the thymidylate synthase gene, the expression of thymidylate synthase mRNA and the microsatellite instability phenotype of colorectal cancer. Oncol Rep. 2004;11:839-43.

24. Vogelstein B, Fearon ER, Kern SE, Hamilton SR, Preisinger AC, Nakamura Y, et al. Allelotype of colorectal carcinomas. Science. 1989;244:207-11.

25. Brody JR, Hucl T, Gallmeier E, Winter JM, Kern SE, Murphy KM. Genomic copy number changes affecting the thymidylate synthase (TYMS) gene in cancer: a model for patient classification to aid fluoropyrimidine therapy. Cancer Res. 2006;66:9369-73.

26. Hubner RA, Liu JF, Sellick GS, Logan RF, Houlston RS, Muir KR. Thymidylate synthase polymorphisms, folate and B-vitamin intake, and risk of colorectal adenoma. Br J Cancer. 2007;97:1449-56.

27. Fernandez-Contreras ME, Sanchez-Hernandez JJ, Gonzalez E, Herraez B, Dominguez I, Lozano $\mathrm{M}$, et al. Combination of polymorphisms within $5^{\prime}$ and $3^{\prime}$ untranslated regions of thymidylate synthase gene modulates survival in 5 fluorouracil-treated colorectal cancer patients. Int J Oncol. 2009;34:219-29.

28. Graziano F, Ruzzo A, Loupakis F, Santini D, Catalano V, Canestrari E, et al. Liver-only metastatic colorectal cancer patients and thymidylate synthase polymorphisms for predicting response to 5fluorouracil-based chemotherapy. Br J Cancer. 2008;99:716-21.

29. Lurje G, Zhang W, Yang D, Groshen S, Hendifar AE, Husain H, et al. Thymidylate synthase haplotype is associated with tumor recurrence in stage II and stage III colon cancer. Pharmacogenet Genomics. 2008;18:161-8.

30. Curtin K, Ulrich CM, Samowitz WS, Bigler J, Caan B, Potter JD, et al. Thymidylate synthase polymorphisms and colon cancer: associations with tumor stage, tumor characteristics and survival. Int J Cancer. 2007;120:2226-32.

31. Hitre E, Budai B, Adleff V, Czegledi F, Horvath Z, Gyergyay F, et al. Influence of thymidylate synthase gene polymorphisms on the survival of colorectal cancer patients receiving adjuvant 5fluorouracil. Pharmacogenet Genomics. 2005;15:723-30.

32. AA ME, del Rio E, Cesar A, Menoyo A, Baiget M. Single nucleotide polymorphism in the $5^{\prime}$ tandem repeat sequences of thymidylate synthase gene predicts for response to fluorouracilbased chemotherapy in advanced colorectal cancer patients. Int $\mathrm{J}$ Cancer. 2004;112:733-7.

33. Park CM, Lee WY, Chun HK, Cho YB, Yun HR, Heo JS, et al. Relationship of polymorphism of the tandem repeat sequence in 
the thymidylate synthase gene and the survival of stage III colorectal cancer patients receiving adjuvant 5-flurouracil-based chemotherapy. J Surg Oncol. 2009;101:22-7.

34. Schwarzenbach H, Goekkurt E, Pantel K, Aust DE, Stoehlmacher J. Molecular analysis of the polymorphisms of thymidylate synthase on cell-free circulating DNA in blood of patients with advanced colorectal carcinoma. Int J Cancer (2009).

35. Gosens MJ, Moerland E, Lemmens VP, Rutten HT, Tan-Go I, van den Brule AJ. Thymidylate synthase genotyping is more predictive for therapy response than immunohistochemistry in patients with colon cancer. Int J Cancer. 2008;123:1941-9.

36. Longley DB, Harkin DP, Johnston PG. 5-fluorouracil: mechanisms of action and clinical strategies. Nat Rev Cancer. 2003;3:330-8.

37. Shirasaka T, Shimamoto Y, Fukushima M. Inhibition by oxonic acid of gastrointestinal toxicity of 5-fluorouracil without loss of its antitumor activity in rats. Cancer Res. 1993;53:4004-9.

38. Sanada Y, Yoshida K, Hihara J, Okada M. Expression of orotate phosphoribosyltransferase in colorectal carcinoma: an immunohistochemical analysis in several components of neoplastic lesions. Oncol Rep. 2008;20:1005-11.

39. Tokunaga Y, Sasaki H, Saito T. Clinical role of orotate phosphoribosyl transferase and dihydropyrimidine dehydrogenase in colorectal cancer treated with postoperative fluoropyrimidine. Surgery. 2007;141:346-53.

40. Suchi M, Mizuno H, Kawai Y, Tsuboi T, Sumi S, Okajima K, et al. Molecular cloning of the human UMP synthase gene and characterization of point mutations in two hereditary orotic aciduria families. Am J Hum Genet. 1997;60:525-39.

41. Ichikawa W, Takahashi T, Suto K, Sasaki Y, Hirayama R. Orotate phosphoribosyltransferase gene polymorphism predicts toxicity in patients treated with bolus 5-fluorouracil regimen. Clin Cancer Res. 2006;12:3928-34.

42. Kitajima M, Takita N, Hata M, Maeda T, Sakamoto K, Kamano T, et al. The relationship between 5-fluorouracil sensitivity and single nucleotide polymorphisms of the orotate phosphoribosyl transferase gene in colorectal cancer. Oncol Rep. 2006;15:161-5.

43. Fleming RA, Milano G, Thyss A, Etienne MC, Renee N, Schneider M, et al. Correlation between dihydropyrimidine dehydrogenase activity in peripheral mononuclear cells and systemic clearance of fluorouracil in cancer patients. Cancer Res. 1992;52:2899-902.

44. Harris BE, Carpenter JT, Diasio RB. Severe 5-fluorouracil toxicity secondary to dihydropyrimidine dehydrogenase deficiency. A potentially more common pharmacogenetic syndrome. Cancer. 1991;68:499-501.

45. Zhang H, Li YM, Jin X. DPYD*5 gene mutation contributes to the reduced DPYD enzyme activity and chemotherapeutic toxicity of 5-FU: results from genotyping study on 75 gastric carcinoma and colon carcinoma patients. Med Oncol. 2007;24:251-8.

46. Ciaparrone M, Quirino M, Schinzari G, Zannoni G, Corsi DC, Vecchio FM, et al. Predictive role of thymidylate synthase, dihydropyrimidine dehydrogenase and thymidine phosphorylase expression in colorectal cancer patients receiving adjuvant 5fluorouracil. Oncology. 2006;70:366-77.

47. Soong R, Shah N, Salto-Tellez M, Tai BC, Soo RA, Han HC, et al. Prognostic significance of thymidylate synthase, dihydropyrimidine dehydrogenase and thymidine phosphorylase protein expression in colorectal cancer patients treated with or without 5-fluorouracil-based chemotherapy. Ann Oncol. 2008;19:915-9.

48. Westra JL, Hollema H, Schaapveld M, Platteel I, Oien KA, Keith $\mathrm{WN}$, et al. Predictive value of thymidylate synthase and dihydropyrimidine dehydrogenase protein expression on survival in adjuvantly treated stage III colon cancer patients. Ann Oncol. 2005;16:1646-53.

49. Meropol NJ, Gold PJ, Diasio RB, Andria M, Dhami M, Godfrey $\mathrm{T}$, et al. Thymidine phosphorylase expression is associated with response to capecitabine plus irinotecan in patients with metastatic colorectal cancer. J Clin Oncol. 2006;24:4069-77.

50. Vallbohmer D, Kuramochi H, Shimizu D, Danenberg KD, Lindebjerg J, Nielsen $\mathrm{JN}$, et al. Molecular factors of 5fluorouracil metabolism in colorectal cancer: analysis of primary tumor and lymph node metastasis. Int J Oncol. 2006;28:527-33.

51. Van Kuilenburg AB, Meinsma R, Zoetekouw L, Van Gennip AH. High prevalence of the IVS14 + 1G> A mutation in the dihydropyrimidine dehydrogenase gene of patients with severe 5-fluorouracilassociated toxicity. Pharmacogenetics. 2002;12:555-8.

52. Morel A, Boisdron-Celle M, Fey L, Soulie P, Craipeau MC, Traore S, et al. Clinical relevance of different dihydropyrimidine dehydrogenase gene single nucleotide polymorphisms on 5fluorouracil tolerance. Mol Cancer Ther. 2006;5:2895-904.

53. Capitain O, Boisdron-Celle M, Poirier AL, Abadie-Lacourtoisie S, Morel A, Gamelin E. The influence of fluorouracil outcome parameters on tolerance and efficacy in patients with advanced colorectal cancer. Pharmacogenomics J. 2008;8:256-67.

54. Amstutz U, Farese S, Aebi S, Largiader CR. Dihydropyrimidine dehydrogenase gene variation and severe 5-fluorouracil toxicity: a haplotype assessment. Pharmacogenomics. 2009;10:931-44.

55. van Kuilenburg $\mathrm{AB}$. Dihydropyrimidine dehydrogenase and the efficacy and toxicity of 5-fluorouracil. Eur J Cancer. 2004;40:939-50.

56. Stankova J, Shang J, Rozen R. Antisense inhibition of methylenetetrahydrofolate reductase reduces cancer cell survival in vitro and tumor growth in vivo. Clin Cancer Res. 2005;11:2047-52.

57. Frosst P, Blom HJ, Milos R, Goyette P, Sheppard CA, Matthews $\mathrm{RG}$, et al. A candidate genetic risk factor for vascular disease: a common mutation in methylenetetrahydrofolate reductase. Nat Genet. 1995; 10:111-3.

58. van der Put NM, Gabreels F, Stevens EM, Smeitink JA, Trijbels FJ, Eskes TK, et al. A second common mutation in the methylenetetrahydrofolate reductase gene: an additional risk factor for neuraltube defects? Am J Hum Genet. 1998;62:1044-51.

59. De Mattia E, Toffoli G. C677T and A1298C MTHFR polymorphisms, a challenge for antifolate and fluoropyrimidine-based therapy personalisation. Eur J Cancer. 2009;45:1333-51.

60. Fernandez-Peralta AM, Daimiel L, Nejda N, Iglesias D, Medina Arana V, Gonzalez-Aguilera JJ. Association of polymorphisms MTHFR C677T and A1298C with risk of colorectal cancer, genetic and epigenetic characteristic of tumors, and response to chemotherapy. Int J Colorectal Dis. 2010;25:141-51.

61. Zhang W, Press OA, Haiman CA, Yang DY, Gordon MA, Fazzone $\mathrm{W}$, et al. Association of methylenetetrahydrofolate reductase gene polymorphisms and sex-specific survival in patients with metastatic colon cancer. J Clin Oncol. 2007;25:3726-31.

62. Pare L, Salazar J, del Rio E, Baiget M, Altes A, Marcuello E, et al. Methylenetetrahydrofolate reductase gene polymorphisms: genomic predictors of clinical response to fluoropyrimidinebased chemotherapy in females. J Clin Oncol. 2008;26:3468. author reply 3468-3469.

63. Etienne MC, Formento JL, Chazal M, Francoual M, Magne N, Formento $\mathrm{P}$, et al. Methylenetetrahydrofolate reductase gene polymorphisms and response to fluorouracil-based treatment in advanced colorectal cancer patients. Pharmacogenetics. 2004;14:785-92.

64. Jakobsen A, Nielsen JN, Gyldenkerne N, Lindeberg J. Thymidylate synthase and methylenetetrahydrofolate reductase gene polymorphism in normal tissue as predictors of fluorouracil sensitivity. J Clin Oncol. 2005;23:1365-9.

65. Chua W, Goldstein D, Lee CK, Dhillon H, Michael M, Mitchell P, et al. Molecular markers of response and toxicity to FOLFOX chemotherapy in metastatic colorectal cancer. $\mathrm{Br} \mathrm{J}$ Cancer. 2009;101:998-1004.

66. Derwinger K, Wettergren Y, Odin E, Carlsson G, Gustavsson B. A study of the MTHFR gene polymorphism C677T in colorectal cancer. Clin Colorectal Cancer. 2009;8:43-8. 
67. Ruzzo A, Graziano F, Loupakis F, Rulli E, Canestrari E, Santini $\mathrm{D}$, et al. Pharmacogenetic profiling in patients with advanced colorectal cancer treated with first-line FOLFOX-4 chemotherapy. J Clin Oncol. 2007;25:1247-54.

68. Zintzaras E, Ziogas DC, Kitsios GD, Papathanasiou AA, Lau J, Raman G. MTHFR gene polymorphisms and response to chemotherapy in colorectal cancer: a meta-analysis. Pharmacogenomics. 2009;10:1285-94.

69. Afzal S, Jensen SA, Vainer B, Vogel U, Matsen JP, Sorensen JB, et al. MTHFR polymorphisms and 5-FU-based adjuvant chemotherapy in colorectal cancer. Ann Oncol. 2009;20:1660-6.

70. Marcuello E, Altes A, Menoyo A, Rio ED, Baiget M. Methylenetetrahydrofolate reductase gene polymorphisms: genomic predictors of clinical response to fluoropyrimidine-based chemotherapy? Cancer Chemother Pharmacol. 2006;57:835-40.

71. Watson MA, Stewart RK, Smith GB, Massey TE, Bell DA. Human glutathione S-transferase P1 polymorphisms: relationship to lung tissue enzyme activity and population frequency distribution. Carcinogenesis. 1998;19:275-80.

72. Romero RZ, Morales R, Garcia F, Huarriz M, Bandres E, De la Haba J, et al. Potential application of GSTT1-null genotype in predicting toxicity associated to 5-fluouracil irinotecan and leucovorin regimen in advanced stage colorectal cancer patients. Oncol Rep. 2006;16:497-503.

73. Stoehlmacher J, Park DJ, Zhang W, Yang D, Groshen S, Zahedy $\mathrm{S}$, et al. A multivariate analysis of genomic polymorphisms: prediction of clinical outcome to 5-FU/oxaliplatin combination chemotherapy in refractory colorectal cancer. $\mathrm{Br} \mathrm{J}$ Cancer. 2004;91:344-54.

74. Stoehlmacher J, Park DJ, Zhang W, Groshen S, Tsao-Wei DD, Yu $\mathrm{MC}$, et al. Association between glutathione S-transferase P1, T1, and M1 genetic polymorphism and survival of patients with metastatic colorectal cancer. J Natl Cancer Inst. 2002;94:936-42.

75. Braun MS, Richman SD, Thompson L, Daly CL, Meade AM, Adlard JW, et al. Association of molecular markers with toxicity outcomes in a randomized trial of chemotherapy for advanced colorectal cancer: the FOCUS trial. J Clin Oncol. 2009;27:551928.

76. Huang MY, Fang WY, Lee SC, Cheng TL, Wang JY, Lin SR. ERCC2 2251A $>$ C genetic polymorphism was highly correlated with early relapse in high-risk stage II and stage III colorectal cancer patients: a preliminary study. BMC Cancer. 2008;8:50. 